\title{
Learning curve of laparoscopic nephrectomy: a prospective pilot study
}

\author{
Mohamed Masoud ${ }^{1 \dagger}$, Ahmed Ibrahim ${ }^{2^{*}}$, Abdelbaset Elemam¹, Adel Elatreisy', Yasser Noureldin³, \\ Mélanie Aubé ${ }^{2}$ and Nader Fahmy ${ }^{2}$
}

\begin{abstract}
Background: Learning curve of laparoscopic nephrectomy (LN) is mainly affected by two main factors: plotting performance and experience. However, there is paucity in the literature addressing the number of cases required to adopt LN. Herein, we aimed to assess the learning curve of $L N$ for various renal disorders and number of cases required to adopt the technique. Between September 2015 and December 2017, consecutive patients undergoing LN for various renal diseases were enrolled in this study. Patients were divided into two groups, the first 20 cases (group A) and subsequent 20 cases (group B). All procedures were performed by a single trainee urologist under supervision of an expert endourologist. Learning curve was assessed using operative time and incidence of complications.

Results: A total of 40 patients were included in this pilot clinical study. Mean age was $38.2 \pm 16.3$ years. The mean operative time for patients in group B was significantly lower than the mean operative time for patients in group A (108.5 vs. $139.3 \mathrm{~min}, p<0.05)$. However, there were no significant differences between both groups in terms of intraoperative blood loss ( 86 vs. $104 \mathrm{ml} ; p=0.081$ ), conversion to open surgery ( $5 \%$ vs. $10 \% ; p=0.256$ ) and postoperative complications ( $5 \%$ vs. $15 \% ; p=0.09$ ) for group B and group A, respectively. Similarly, there was no significant difference between both groups in terms of hospital stay ( $42 \pm 8 \mathrm{vs} .46 \pm 11 \mathrm{~h} p=0.01)$. The trainee surgeon reached a plateau after 22 cases.
\end{abstract}

Conclusions: Our study suggests that a minimum of $22 \mathrm{LN}$ procedures are needed in order to adopt the technique of laparoscopic nephrectomy. Learning curve of $L N$ is mainly affected by number of performed procedures within a short period of time.

Keywords: Laparoscopy, Nephrectomy, Simulators, Learning curve, E-BLUS

\section{Background}

Since it was initially proposed by Ralph Clayman in 1991, laparoscopic nephrectomy (LN) for benign and malignant renal tumors has been adopted and supported by long-term data on safety and efficacy [1,2]. Consequently, it has been accepted as a standard treatment in both developing and developed countries [3].

\footnotetext{
*Correspondence: ahmed.i.ibrahim@mail.mcgill.ca

${ }^{\dagger}$ Mohamed Masoud and Ahmed Ibrahim are co-first authors and contributed equally.

${ }^{2}$ Division of Urology, Department of Surgery, McGill University Health

Center, Montreal, QC, Canada

Full list of author information is available at the end of the article
}

There are several advantages for laparoscopic nephrectomy when compared with the regular open approach: first, better visualization due to magnified field. Second, shorter hospital stays and better cosmetic results. The initial limitation of longer operative times and greater costs is now considered less prominent factors due to wide adoption and increasing experience of this technique [4].

It is intuitive that repetitive performance of a certain task over short period of time would definitely increase the experience of the surgeon and subsequently reduce the surgical complications. Learning curve is mainly affected by two main factors, plotting performance and experience (number of performed tasks). Several studies 
demonstrated that a certain number of cases are required to adopt the technique and reduce surgical complications [5-8]. We hypothesized that laparoscopic training at simulator-based environment in addition to both dry and wet laboratory would reduce the number of cases required to adopt laparoscopic nephrectomy and reduce the technical complications. Therefore, the aim of the present study was to assess the learning curve of laparoscopic nephrectomy and number of cases required to adopt LN technique in a cohort of trainee urologist who has access to laparoscopic simulation training.

\section{Methods}

After obtaining local institutional ethics approval, between September 2015 and December 2017, 40 consecutive patients undergoing laparoscopic nephrectomy (LN) for various renal diseases (malignant and nonmalignant) were assessed in a prospective fashion. Inclusion criteria were benign renal diseases, non-functioning kidney-either atrophic or hydronephrotic documented by nuclear isotope scanning-and malignant renal diseases diagnosed by radiologic studies. Exclusion criteria were patients with multiple comorbidities such as congestive heart failure, respiratory insufficiency, patient with uncorrectable coagulopathy, morbid obesity (BMI'35), abdominal wall infection, aortic aneurysms, acute diffuse peritonitis and presence of distended bowel. Patients were divided into two groups: The first 20 cases were included in group A and the next 20 cases were included in group B. All procedures were performed by single trainee urologist under supervision of expert, fellowship-trained, endourologist. The urology trainee was enrolled in a training program with mandatory laparoscopic simulation skills training, using both dry laboratory (laparoscopic training box) once weekly and wet laboratory simulation training (using a live pig training model) biannually. Surgicaltechnique used for training was based on the validated basic laparoscopic urologic surgery (BLUS) technique which is proposed by the AUA [9]. Learning curve was assessed in terms of operative time and the incidence of complications.

\subsection{Technique of the procedure}

Trans-peritoneal LN is typically performed using either a three- or four-port technique.

The technique of LN has been previously described [3]. In brief, the intraperitoneal space was accessed through closed technique using a Veress needle in all cases. After pneumoperitoneum is achieved, the remaining ports are placed under direct vision. The colon is reflected along Toldt's line to expose the kidney. The renal hilum is identified at the level of the aorta after the lower pole of the kidney is lifted away from the psoas muscle. The upper pole of the kidney is dissected away from the liver on the right side, or the spleen and the tail of the pancreas on the left. The renal artery is occluded and transected with clips-either titanium or Hem-o-lok (Weck Closure Systems, Research Triangle Park, NC). The renal vein is then occluded and transected with hem-o-lok clips or ligated with a silk suture (Fig. 1).

If indicated, the adrenal gland is resected by securing the adrenal vein with titanium clips, and the remainder of the kidney can be mobilized bluntly.

The renal specimen is then retrieved through a small incision and sent for histopathological examination.

\subsection{Outcome measures}

Operative efficacy measures include laparoscopic time (time started from introduction of the laparoscopy into the abdomen until extraction of kidney tissue).

Operative safety measures include intraoperative blood loss and bleeding necessitating blood transfusion or conversion to open surgery. Moreover, perioperative hemoglobin and hematocrit levels were noted. Length of hospital stay and perioperative complications was also noted.

Postoperative follow-up patients were scheduled for 6 months after surgery for any specific complications related to the procedure.

\subsection{Statistical analysis}

Data were collected and tabulated using SPSS software version 21 (SPSS Inc., Chicago, IL, USA). Descriptive statistics were presented in terms of percentages, frequencies and means. Differences between both groups were compared with the Fisher's exact test for categorical variables and Student's $t$-test or Mann-Whitney $U$ test to compare normally and abnormally distributed continuous variables, respectively. Two-tailed $p$ value of less than 0.05 was considered significant.

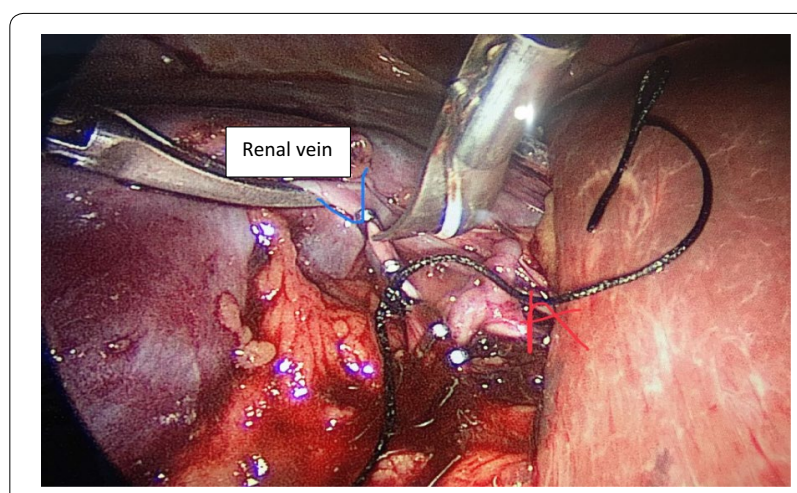

Fig. 1 Identification of the renal pedicle, clamping and cutting using laparoscopic scissor 


\section{Results}

A total of 40 patients were included in this pilot clinical study. The mean age was $38.2 \pm 16.3$ years while the mean body mass index (BMI) was $28.5 \pm 4.2 \mathrm{~kg} / \mathrm{m}^{2}$. Twenty-seven patients (67.5\%) underwent left-sided LN.

Regarding the learning curve, it was found that the mean operative time for patients in group B was significantly lower than the mean operative time for group A patients (108.5 vs. $139.3 \mathrm{~min}, p=0.01)$. The mean operative time decreased by $21.5 \%$ (30 $\mathrm{min}$ ) in group $B$ (Fig. 2). The surgeon reached a plateau in operative time after 22 cases (Fig. 3).

Intraoperative blood loss decreased from $104 \mathrm{cc}$ with group A to $86 \mathrm{cc}$ with group B. However, this difference was not statistically significant $(p=0.081)$. Similarly, rate of conversion to open surgery decreased from two patients $(10 \%)$ in group A to one patient (5\%) in group B $(p=0.256)$. No significant vascular injuries occurred during this study.

With regard to postoperative complications, there was no significant difference between both groups (15\% in group A vs. $5 \%$ in group $\mathrm{B} ; p=0.09$ ). In group $\mathrm{A}$, two cases $(10 \%)$ had an early postoperative complication ( $<30$ days), one of them developed scrotal ecchymosis (managed conservatively), and the other one developed fever on postoperative day one after right-sided LN for xanthogranulomatous pyelonephritis (XPS) which also managed conservatively. One patient (5\%) from group A developed port-site hernia ( $>30$ days) managed by surgical repair (Table 1). Similarly, there was no significant difference between both groups in terms of length of hospital stay ( $42 \pm 8$ vs. $46 \pm 11 \mathrm{~h}, p=0.121)$. Postoperative pathology showed that the majority of cases were due to obstructive renal atrophy (52.5\%) (Table 2).

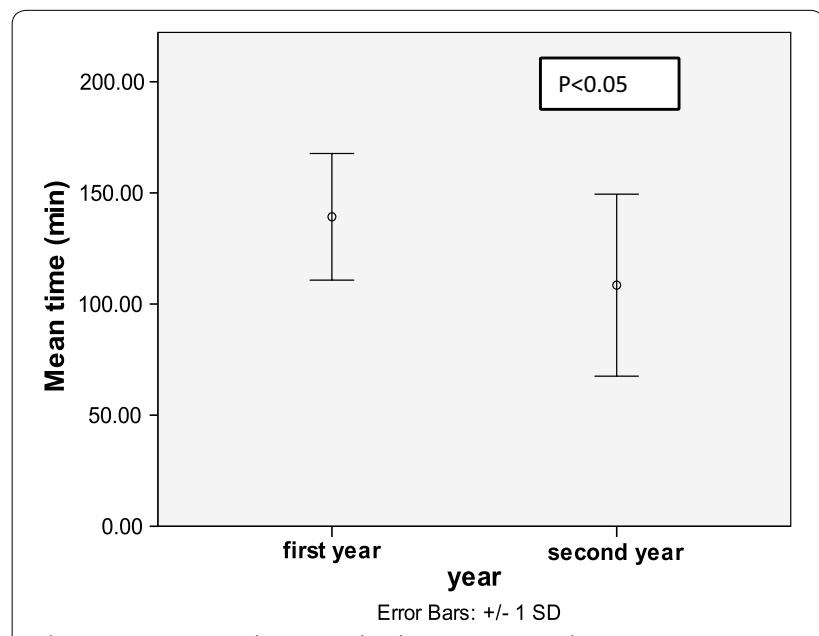

Fig. 2 Comparison between both groups regarding operative time

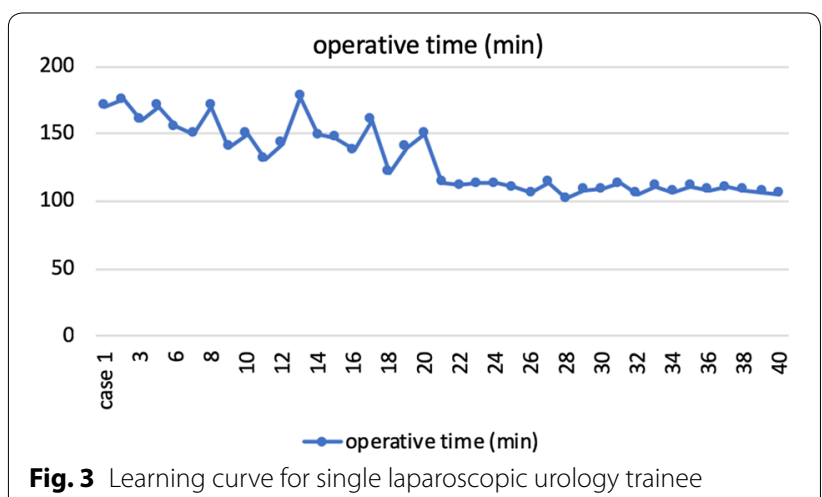

\section{Discussion}

Laparoscopic skills evolve with repetition, and it is associated with slow learning curve skills [2]. Some authors reported that at least 50 cases were required to adopt the technique of LN and they reported significant improvement in the outcome and decrease in complication rate

\section{Table 1 Patients' demographics, intraoperative and postoperative data}

\begin{tabular}{ll}
\hline Parameter & Value \\
\hline Mean age \pm SD (years) & $38.2 \pm 16.3$ \\
Gender, N (\%) & \\
Male & $24) 60 \%)$ \\
Female & $16(40 \%)$ \\
Mean BMI (Kg/M) & $28.58 \pm 4.2$ \\
Indication of nephrectomy, N (\%) & \\
Non-functioning, loin pain & $21(52.5 \%)$ \\
Non-functioning, recurrent UTI & $14(35 \%)$ \\
Upper urinary tract tumor & $5(12.5 \%)$ \\
Side of the procedure & \\
Left nephrectomy & $27(67.5 \%)$ \\
Right nephrectomy & $13(32.5 \%)$ \\
Laparoscopy time & $123.5 \pm 49.7$ \\
Procedure time & $148.7 \pm 32.4$ \\
Perioperative complications & \\
Scrotal ecchymosis & One case (5\%) \\
Fever & One case (5\%) \\
Port-site hernia & One case (5\%) \\
Hospital stay (h) & $44 \pm 78$ \\
Postoperative histopathology & \\
Obstructive atrophy & \\
Chronic pyelonephritic & $21(52.5 \%)$ \\
Xanthogranulomatous pyelonephritis & $10(25 \%)$ \\
Renal tuberculosis & $3(7.5 \%)$ \\
Upper urinary tract TCC & $1(2.5 \%)$ \\
\hline BMIbody mas index,Nnumber & $5(12.5 \%)$ \\
\hline
\end{tabular}

$B M I$ body mass index, $N$ number of patients, UTI urinary tract infection, TCC transitional cell carcinoma 


\begin{tabular}{|c|c|c|c|c|c|}
\hline & \multicolumn{4}{|c|}{ Group } & \multirow[t]{3}{*}{$p$ value } \\
\hline & \multicolumn{2}{|c|}{$\begin{array}{l}\text { Group } \\
\mathrm{A}(n=20)\end{array}$} & \multicolumn{2}{|c|}{$\begin{array}{l}\text { Group B } \\
(n=20)\end{array}$} & \\
\hline & $N$ & $\%$ & $N$ & $\%$ & \\
\hline \multicolumn{6}{|l|}{ Early complications } \\
\hline No & 18 & 90 & 20 & 100 & 0.49 \\
\hline Scrotal ecchymosis & 1 & 5 & 0 & 0 & \\
\hline Fever & 1 & 5 & 0 & 0 & \\
\hline \multicolumn{6}{|l|}{ Follow-up } \\
\hline Number of complications & 19 & 95 & 20 & 100 & 1 \\
\hline Port-site hernia & 1 & 5 & 0 & 0 & \\
\hline
\end{tabular}

after the initial 50 cases [3]. However, these series were published early after introducing the LN technique. Currently, with the development of technology and simulation-based training, surgeons can improve and practice their skills prior to operating on patients under supervision. They can also receive detailed feedback about their performance during procedures. This has led to continuous improvement in surgical skills and patient outcomes. Consequently, we hypothesized that laparoscopic training at simulator-based environment in addition to both dry and wet laboratory would reduce the number of cases required to adopt laparoscopic nephrectomy and reduce the technical complications. Therefore, the aim of the present study was to assess the learning curve of laparoscopic nephrectomy after performing validated basic laparoscopic urologic surgery (BLUS) which was proposed by the AUA [9]. In addition, the trainee was trained to do laparoscopic nephrectomy in the animal lap.

In the present study, for better evaluation of the learning curve, the cohort was divided into two groups: the early twenty cases (group A) and the next twenty cases (group B). It was found that the mean laparoscopy time in group A was 139.3 min which was dropped to 108.5 min in group B. This was comparable with the second group that was reported by Keeley and Tolley [7]; the operative time in the first 20 cases was $204 \mathrm{~min}$, which reduced to $108 \mathrm{~min}$ in the last 20 cases. However, our first group of cases was substantially less than that reported by Keeley and Tolley (139.3 vs. $204 \mathrm{~min}$ ) which might emphasize the impact of training in dry and wet lap prior operating in the main OR. In another review by Eraky et al. [8], the mean operative time for the initial 53 cases and the subsequent 53 cases was $217 \pm 84 \mathrm{~min}$ and $154 \pm 48 \mathrm{~min}$, respectively. With experience, identification of anatomical landmarks and techniques of dissection became refined which subsequently lead to a significant decrease in the operative time and the amount of blood loss during the surgery which was also found in our study.

Rassweiller et al. [10] observed that the majority of the complications, conversion rates and re-operation rates usually encountered in the first 20 cases of each surgeon during the phase of the initial learning curve. In another review, it was reported that there was a relatively slow learning curve during the first 50 cases [11]. In a study by Gill et al., it was reported that $71 \%$ of the complications occurred during the first 20 patients. In their series, Modi et al., reported $20 \%$ of open surgery conversion in the initial 20 cases. This was attributed to failure to acquire laparoscopic skills and slowly the progression of the surgeons [12, 13]. Some authors explained that the majority of open surgery conversions were attributed to technical failures such as inflammation in the peri-renal region, renal pathology and inexperience of the surgeon [13]. In the present study, conversion to open surgery was encountered in $10 \%$ in group $\mathrm{A}$ and $5 \%$ in group $\mathrm{B}$. This was relatively lower than the previously published series (Table 2). Furthermore, the entire postoperative complications decreased from $15 \%$ in group A to $0 \%$ in group B. In addition, intraoperative blood loss was dropped by about $17.3 \%$, $104 \mathrm{~mL}$ in group A and decrease to $86 \mathrm{~mL}$ in group B. This might emphasize the importance of simulationbased training and animal lap training in adopting the laparoscopic skills and consequently reducing the learning curve and potential operative complications.

Finally, as the surgeon becomes more skilled in laparoscopy, he can manipulate and proceed for more difficult cases of laparoscopic nephrectomy (XGP). Kidneys which were affected by chronic infections, previoussurgery or fibrosis, were found to be difficult to treat laparoscopically. Therefore, they should be done by expert laparoscopic surgeons in order to avoid intraoperative complications [14]. Others reported that with their hands, operative experience did not decrease the complication rate. It was in part due to liberalizing their criteria for patient selection, and as the experience increased, the willingness to operate laparoscopically in cases that are more complex increased [15].

This study is not without its limitations including the relatively small sample size and variability of renal disorders which might be one of the potential confounders that interfere with proper assessment of the learning curve. In addition, the study was done by single trainee; the study may be reproduced with a group of trainees. Nevertheless, this pilot study demonstrated the minimum number of cases required to adopt the LN technique, in addition to the importance of patient selection at the initial phase of the learning curves. 


\section{Conclusions}

Our study suggests that a minimum of 22 LN procedures are needed in order to adopt the technique of laparoscopic nephrectomy. Learning curve of LN might be affected by two main factors: plotting performance and number of performed tasks in a short period of time. Laparoscopic training using dry and wet laboratories could probably reduce the number of procedures required to adopt the technique. However, further studies are warranted to assess transfer.

\section{Abbreviations}

BLUS: basic laparoscopic urologic surgery; BMI: body mass index; LN: laparoscopic nephrectomy; XPS: xanthogranulomatous pyelonephritis.

\section{Acknowledgements}

None.

\section{Authors' contributions}

MM contributed to data collection and drafting the initial manuscript. Al contributed to drafting the initial manuscript and editing the final manuscript. AE contributed to data collection and editing the final manuscript. AE contributed to drafting the initial manuscript. YN contributed to data collection and drafting the initial manuscript. MA contributed to data collection and editing the final manuscript. NF contributed to data collection and editing the final manuscript, senior author. All authors approved the final manuscript as submitted and agree to be accountable for all aspects of the work.

\section{Funding}

None.

\section{Availability of data and materials}

The datasets used and/or analyzed during the current study are available from the corresponding author on reasonable request.

\section{Ethics approval and consent to participate}

From the Scientific Research Ethics Committee of Faculty of Medicine-AlAzher University - which was later approved by other participating centers (reference number is not applicable). An informed written consent was obtained from all participating patients after explaining the aim, adverse events and the methodology of the study.

\section{Consent for publication}

Not applicable.

\section{Competing interests}

The authors declare that they have no competing interests.

\section{Author details}

${ }^{1}$ Department of Urology, Al-Azhar University, Cairo, Egypt. ${ }^{2}$ Division of Urology, Department of Surgery, McGill University Health Center, Montreal, QC, Canada. ${ }^{3}$ Department of Urology, Benha University, Benha, Egypt.
Received: 9 December 2019 Accepted: 29 January 2020

Published online: 22 April 2020

\section{References}

1. Clayman R, Kavoussi L, Soper N et al (1991) Laparoscopic nephrectomy: initial case report. J Urol 146(2):278-282

2. Simon D, Castle P, Ferrigni G et al (2004) Complications of laparoscopic nephrectomy: the Mayo clinic experience. J Urol 171(4):1447-1450

3. Shoma A, Eraky I, El-Kappany H (2001) Laparoscopic nephrectomy: prediction of outcome in relation to preoperative risk factors in two approaches. J Endourol 15(5):517-523

4. Vallancien G, Cathelineau X, Baumert H, Doublet D, Guillonneau B (2002) Complications of transperitoneal laparoscopic surgery in urology: review of 1311 procedures at a single center. J Urol 168(1):23-26

5. Gill I, Meraney A, Schweizer D et al (2001) Laparoscopic radical nephrectomy in 100 patients. Cancer 92(7):1843-1855

6. Gozen A, Gherman V, Akin Y et al (2017) Evaluation of the complications in laparoscopic retroperitoneal radical nephrectomy; an experience of high volume centre. Arch Ital Urol Androl 89(4):266-267

7. Keeley X, Tolley D (1998) A review of our first 100 cases of laparoscopic nephrectomy: defining risk factors for complications. Br J Urol Int 82(5):615-618

8. Eraky I, El-Kappany H, Ghoniem M (1995) Laparoscopic nephrectomy: Mansoura experience with 106 cases. Br J Urol Int 75(3):271-275

9. Kowalewski T, Sweet R, Lendvay T et al (2016) Validation of the AUA BLUS tasks. J Urol 195(4 Pt 1):998-1005

10. Rassweiler J, Fornara P, Weber M (1998) Laparoscopic nephrectomy: the experience of the laparoscopy working group of the German Urological Association. J Urol 160(1):18-21

11. Rassweiler J, Seemann O, Frede T, Henkel TO, Alken P (1998) Retroperitoneoscopy: experience with 200 cases. J Urol 160(4):1265-1269

12. Gill I, Clayman R, McDougall E (1995) Advances in urologic laparoscopy. J Urol 154(4):1275-1294

13. Modi P, Kandam G, Dodia S et al (2005) Laparoscopic retroperitoneal nephrectomy: overcoming the learning curves. Indian J Urol 21:102-110

14. Kuo L, Siqueira M, Shalbav L (2003) Laparoscopic simple nephrectomy transperitoneal and retroperitoneal approaches. In: Nakada SY (ed) Essential urologic laparoscopy: the complete clinical guide. Human Press. Inc., Totowa, p 79

15. Seifman B, Dunn R, Wolf J (2003) Transperitoneal laparoscopy into the previously operated abdomen: effect on operative time, length of stay and complications. J Urol 169(1):36-40

\section{Publisher's Note}

Springer Nature remains neutral with regard to jurisdictional claims in published maps and institutional affiliations.

\section{Submit your manuscript to a SpringerOpen ${ }^{\circ}$ journal and benefit from:}

- Convenient online submission

- Rigorous peer review

- Open access: articles freely available online

- High visibility within the field

- Retaining the copyright to your article

Submit your next manuscript at springeropen.com 